\title{
Maffucci Syndrome Complicated by Giant Chondrosarcoma in the Left Ankle with an IDH1 R132C Mutation: Case Report
}

\section{Haiyan Lv}

Taizhou Hospital of Zhejiang Province

Hantao Jiang

Taizhou Hospital of Zhejiang Province

Minge Zhang

Taizhou Hospital of Zhejiang Province

Huarong Luo

Taizhou Hospital of Zhejiang Province

Zhenghua Hong

Taizhou Hospital of Zhejiang Province

Hai Yang

Taizhou Hospital of Zhejiang Province

Weiming $\mathrm{Xu}$

Taizhou Hospital of Zhejiang Province

\section{Bo Shen}

Taizhou Hospital of Zhejiang Province

Rangteng Zhu ( $\nabla$ tzlqzit@126.com )

Taizhou Hospital of Zhejiang Province https://orcid.org/0000-0002-5651-1454

\section{Case report}

Keywords: Maffucci syndrome, chondrosarcoma, isocitrate dehydrogenase

Posted Date: October 25th, 2021

DOI: https://doi.org/10.21203/rs.3.rs-771590/v2

License: (c) (1) This work is licensed under a Creative Commons Attribution 4.0 International License. Read Full License 


\section{Abstract}

\section{Background}

Maffucci syndrome is a rare, nonhereditary congenital mesodermal dysplasia characterized by multiple enchondromas and hemangiomas. It is associated with an increased risk of the development of malignant tumors. We present a case of 45-year-old man with Maffucci syndrome to supplement the clinical manifestations and explore the molecular mechanism of Maffucci syndrome.

Case presentation

The patient was underwent amputation surgery to inhibit tumor development and diagnosed as Maffucci syndrome with 1-2 grade giant chondrosarcoma in the left ankle. In addition, the whole exon analysis by Next Generation Sequencing revealed isocitrate dehydrogenase 1 R132C mutation in chondrosarcoma lesions but not in blood DNA.

\section{Conclusions}

This case report presents the genetic evidence for the inclusion of chondrosarcoma among tumors characterizing Maffucci syndrome. Consequently, it is suggested that the patient with Maffucci syndrome should be followed up more actively to exclude neoplasms due to IDH1 R132C somatic mutation.

\section{Background}

Maffucci syndrome (MS) is a rare cartilage dysplasia syndrome. In 1881, Angelo Maffucci first described a non-genetic disease characterized by multiple hemangiomas and endogenous chondroma during adolescence[1]. Clinically, MS is distinguished from Ollier disease (OD) by identifying soft-tissue vascular lesions accompanying MS but not OD. MS patients usually have asymmetric skeletal deformities and limb length differences in the first decade of life and may need surgery. However, Additional tumors have been reported in MS patients with the disease progression, including lymphangiomas, glioma, acute myeloid leukemia, and ovarian fibrosarcoma[2, 3]. Recently, it was revealed that individuals with MS and OD harbor somatic mosaicism of mutations in isocitrate dehydrogenase 1 (IDH1) or isocitrate dehydrogenase 2 (IDH2), as the crucial factors in the pathogenesis of these diseases[4].

At present, there are few studies on MS with chondrosarcoma and detailed gene analysis. In this article, we report a case of giant chondrosarcoma secondary to MS with a long medical history. Moreover, we conducted whole-exome sequencing of germline DNA and chondrosarcoma tissue by Next Generation Sequencing (NGS) to further explore the pathogenesis of MS.

\section{Case Presentation}

\section{Medical history}


A 45-year-old male patient was admitted to our hospital because of a lump in the left ankle for 20 years and enlarging for the last two years. From the appearance of the lump to the subsequent 18 years, there were no significant changes. However, the left ankle lump began to enlarge rapidly to about $40 \mathrm{~cm}$ in diameter and then developed skin ulceration with apparent purulent discharge two years ago.

At the age of 7 , the patient accidentally found several soybean-sized soft lumps in two feet without pain and dysfunction, which was not paid attention to at that time. In the next few years, the patient developed multiple lumps in his limbs. When the patient was 19 years old, he was finally diagnosed with Maffucci Syndrome for the extremities with multiple angiomatosis and enchondromas and underwent resection surgery in our hospital. However, the symptoms reoccurred at the original resection site only two years later, and resection surgery again. Confusingly, reoccurrence happened soon after the second resection surgery.

\section{Physical and imaging examination}

After the patient was admitted in 2020 , the physical examination showed multiple angiomatoses and enchondromas in the extremities (Figure 1A, B), and a giant lump of about $35 \mathrm{~cm} \times 30 \mathrm{~cm} \times 30 \mathrm{~cm}$ in the left ankle, with local skin ulceration and evident purulent secretions discharge (Figure 1C, D). There was a big lump on the right scapula without tenderness or percussion pain (Figure 1E).

X-ray examination of both hands revealed changes in bone morphology and density of both hands, including spherical expansive bone destruction in the right thumb and multiple phleboliths in the hands and wrists (Figure 2A, B). In addition, it was also found bone density and morphological changes of the left ilium and left femur (Figure 2C), the flexion deformity of the left knee joint, multiple venous stones around the left knee joint (Figure 2D). Moreover, X-ray examination showed local spherical expansive bone destruction with multiple calcifications at the left tarsal, multiple chondromatosis with bone deformity, and multiple hemangiomas (Figure 2E, F). Moreover, the magnetic resonance imaging (MRI) examination of the left femur of the patient was performed to determine the amputation plane (Figure 3).

\section{Laboratory examination}

$C$ reactive protein ( $C R P$ ) and erythrocyte sedimentation rate ( ESR ) were consistently higher than the critical value during hospitalization (Figure 4A, B). The number of white blood cells increased sharply from admission to operation and decreased gradually after the operation (Figure 4C). The alkaline phosphatase (ALP) level was significantly higher than the critical value, reaching the highest point of 572 $\mathrm{U} / \mathrm{L}$, and gradually decreased after the operation, but did not return to the normal level (Figure 4D). Blood calcium level was below the critical value before operation and gradually returned to normal after the operation (Figure 4E), and blood phosphorus returned to normal level after the operation (Figure 4F). Serum calcium, phosphorus, and ALP levels were normal during hospitalization in 1994 and 1997 (data not shown ). Hemoglobin levels increased gradually from admission to discharge but remained below the critical value (Figure 4G). Alanine aminotransferase (ALT), aspartate aminotransferase (AST) and 
gamma-glutamyltransferase ( $\mathrm{Y}-\mathrm{GGT})$ were abnormal and tended to be normal during the hospitalization (Figure 4H).

\section{Pathological examination}

The chondrosarcoma of the left ankle was assessed as grade 1-2 by pathological diagnosis. Multiple cavernous hemangiomas with thrombosis were in the left lower limb (Figure 5A). Enchondroma could be seen in the tibia. The cells were loose, with less atypia (Figure 5B). Besides, chondrosarcoma invades surrounding soft tissues, and necrosis was seen in some areas(Figure $5 \mathrm{C}$ ). Chondrosarcoma cells with prominent atypia and visible nuclear fission (Figure 5D).

\section{Gene examination}

NGS was applied to detect the whole exon group of peripheral blood germline DNA and chondrosarcoma tissue of the patient. For blood DNA, genome GRCh37/hg19 was chosen as the reference. We performed high-throughput detection and analysis of 20,000 genetic disease gene combination exons and their adjacent $\pm 10 \mathrm{bp}$ introns of the subjects including AARS, ANO5, CAPN3, etc., and focused on the genes related to the blood system, bone, and hereditary tumors. No pathogenic variation, suspected pathogenic variation, and unknown clinical significance related to the clinical manifestations of the subjects were detected, but the possibility of other known and unknown pathogenic variation outside the scope of this gene detection was not excluded.

While for chondrosarcoma tissue, blood DNA sequence was selected as the reference. The detection included ALK, BRAF, EGFR, ERBB2, KRAS, NRAS, MET, KIT, PIK3CA, and other tumor diagnosis and treatment-related genes. The sequencing results showed that the amino acid encoded by the 132nd codon of the IDH1 gene changed from arginine to cysteine, namely IDH1 R132 C mutation, and the mutation ratio/copy number was $14.71 \%$.

\section{Discussion}

This article reports a case of giant chondrosarcoma secondary to MS. The patient had a 39-year history of MS and a 2-year history of chondrosarcoma in the left ankle. Due to the large size (40 $\mathrm{cm}$ in diameter) and the highly malignant of the left ankle chondrosarcoma, we have to perform an amputation surgery to inhibit the tumor development. The whole exon analysis revealed IDH1 R132C mutation in chondrosarcoma lesions but not in blood DNA, indicating IDH1 somatic mosaic mutation triggers tumorigenesis.

Enchondroma is benign intramedullary chondroma that could occur at any position. In our study, it develops on the bilateral scapula, the right fifth rib, both ilium, the left pubic branch, the left femur, the upper and lower ends of tibia and fibula, and the metacarpal and phalanx. The risk

of enchondroma developing into chondrosarcoma approximately $25 \%$ to $30 \%$ in patients with MS, usually younger than primary chondrosarcoma patients[5]. Malignant changes frequently occur after 40 years old 
and could occur in any part of the affected bone. Pelvic and upper limb bones are more prone to malignant changes. The patient in our study deteriorated to chondrosarcoma in the left ankle around 43 years old. Skull base chondrosarcoma accounted for $5 \%$ to $10 \%$ of all MS reports[6]. Rarely, it has been reportd a 34-year-old man with tracheal chondrosarcoma and a 39-year-old woman with nasal cavity chondrosarcoma in MS patients[7, 8]. Hemangioma can be seen in many parts, including subcutaneous, mucosal surface, visceral organs, respiratory tract, gastrointestinal tract, aorta, etc. Multiple hemangiomas in both hands were shown in this case.

The IDH1 gene encodes intracytoplasmic nicotinamide adenine dinucleotide phosphate-dependent isocitrate dehydrogenase, which can catalyze isocitrate to produce a-ketoglutarate (a-KG). However, the mutant IDH1 catalyzes the reduction of a-KG to 2-hydroxyglutaric acid (2-HG), competitively inhibiting the activity of a-KG-dependent dioxygenase and demethylases[9], resulting in hypermethylation and down-regulation of tumor suppressor[10]. In addition, the mutant IDH1 affects the generation of nicotinamide adenine dinucleotide phosphate (NADPH)[9], which is of great significance in the synthesis of glutathione (GSH). GSH is an essential antioxidant against reactive oxygen species and free radicals, which may also be one of the mechanisms of mutant IDH1 promoting tumor occurrence and development[11].

Literatures showed that $77 \%$ of MS patients carry IDH1 or IDH2 mutations[12]. Moreover, recurrent somatic mutations in IDH1 and IDH2 occurred in about $80 \%$ of patients with grade II-III glioma and secondary glioblastoma (GBM)[13], and $10 \%-20 \%$ of patients with acute myeloid leukemia (AML), with a low incidence in other cancers; the majority of these lesions involve arginine (R) residue mutations in IDH1 codon 132 (IDH1R132), residue 140 and 172 of IDH2 (IDH2 R140 and IDH2 R172)[14, 15]. It is suggested that somatic heterozygous mutations in IDH1 or IDH2 are also crucial in the development of some malignant tumors, and the increased risk of MS associated with other tumors may be due to common genetic background-mosaic mutations in IDH1 or IDH2.

A MS patient with jugular foramen chondrosarcoma and pituitary adenoma revealed the same IDH1R132C mutation in both tumors[16]. Recently, another literature have shown common IDH1R132C mutations in sellar, brainstem, and skull base tumors in MS patients[17]. Up to now, the IDH1 R132C mutation was only present in the ankle chondrosarcoma tissue but not in the blood of this patient, indicating there will be other tumors in the future. Thereby, it is necessary to carry out the longterm follow-up of the patient and actively recommend further examination and diagnosis.

The treatment of MS aims to alleviate the clinical symptoms of patients and detect malignant lesions early. The essential biochemical indicator of IDH1 and IDH2 mutations in peripheral blood is the abnormal increase of the 2-HG level[9], which may be a sensitive and specific predictor. Surgery is the primary treatment for bone disease and vascular disease, and amputation should be considered for patients with severely affected functions or malignant changes. The prognostic challenge of MS not only comes from the skeletal deformities and secondary limb length differences caused by itself, as well as the potential risk of malignant transformation into chondrosarcoma. Researchers conducted on 44 
patients with endogenic chondroma, 7 cases were diagnosed with MS, and 37 cases were diagnosed with Ollier syndrome, no patient died of skeletal sarcoma, but four of five patients with non-skeletal malignancies died in the study[18]. Therefore, in addition to routine clinical care for MS patients, clinicians should actively detect the development of endogenous chondromas in various parts and do well in tumor detection outside the bone tissue, such as the brain and abdomen.

Given the critical role of mutant IDH1, IDH2, and their products in tumorigenesis and progression, another strategy for MS treatment is targeted therapy for mutant enzymes and products. Small molecules AGI5198 and AGI6780 specifically inhibit the enzyme activity of mutant IDH1 and IDH2, which can inhibit the proliferation of glioma cells and leukemia cells, and successfully induced tumor cell differentiation, confirming the feasibility of mutant enzyme inhibitors for targeted treatment of such tumors[19, 20$]$.

\section{Conclusion}

In summary, we have demonstrated a somatic IDH1 R132C mutation in the giant chondrosarcoma of MS patients. To reduce missed diagnosis or early detection of concomitant lesions, imaging examination and follow-up of other organs are essential. Considering that no subsequent shared genetic events were identified in the chondrosarcoma from our limited analyses, it is necessary to conduct a further comprehensive investigation to discover IDH associated tumorigenesis.

\section{Abbreviations}

MS: Maffucci syndrome; OD: Ollier disease; IDH: isocitrate dehydrogenase; NGS: Next Generation Sequencing; MRI: magnetic resonance imaging; CRP: $C$ reactive protein; ESR: erythrocyte sedimentation rate; ALP: alkaline phosphatase; $Y-G G T:$ gamma-glutamyltransferase; a-KG: a-ketoglutarate; 2-HG: 2hydroxyglutaric acid; NADPH: nicotinamide adenine dinucleotide phosphate; GSH: glutathione; GBM: secondary glioblastoma; AML: acute myeloid leukemia; R: arginine

\section{Declarations}

\section{Ethics approval and consent to participate}

Data from the patients were analyzed: Medical Ethics Review Board Taizhou Hospital of Zhejiang Province, K20210718;

\section{Consent for publication}

Consents for publication of the results of physical, imaging, laboratory, pathological and gene examination (Figure 1 to Figure 5) were provided by the patients.

\section{Availability of data and materials}


The datasets used and/or analysed during the current study are available from the corresponding author on reasonable request.

\section{Competing interests}

The authors declare that they have no competing interests.

\section{Funding}

This work was supported by Taizhou Science and Technology Planning Project (21ywb23, 1802ky01).

\section{Authors' contributions}

JHT collected the data. LHY and JHT drafted the manuscript and contributed to all other quality aspects of the study. ZMG and LHR aided in the case report and data processing. ZRT and LHY performed critical revision of the manuscript. All authors read and approved the final manuscript.

\section{Acknowledgements}

Not applicable.

\section{References}

1. Pansuriya TC, Kroon HM, Bovée J. Enchondromatosis: Insights on the different subtypes. International journal of clinical and experimental pathology 2010;3(6):557-69.

2. Auyeung J, Mohanty K, Tayton K. Maffucci lymphangioma syndrome: an unusual variant of Ollier's disease, a case report and a review of the literature. Journal of Pediatric Orthopaedics $B$ 2003;12(2):147-50.

3. Prokopchuk O, Andres S, Becker K, Holzapfel K, Hartmann D, Friess H. Maffucci syndrome and neoplasms: a case report and review of the literature. BMC Research Notes 2016;9(1):126.

4. Amary MF, Damato S, Halai D, Eskandarpour M, erisha FB, onar FB, et al. Ollier disease and Maffucci syndrome are caused by somatic mosaic mutations of IDH1 and IDH2. Nature Genetics 2011;43(12):1262-65.

5. Mahajan AM, Ganvir S, Hazarey V, Mahajan MC. Chondrosarcoma of the maxilla: A case report and review of literature. Journal of Oral and Maxillofacial Pathology 2013;17(2):269-73.

6. Adrianna R, Artur S. Do intracranial neoplasms differ in Ollier disease and maffucci syndrome? An indepth analysis of the literature. Neurosurgery 2009(6):1106-13.

7. Wagnetz U, Patsios D, Darling G, Heras FL, Hwang D. Tracheal chondrosarcoma-a rare complication in Maffucci syndrome. British Journal of Radiology 2009;82(981):178-81.

8. Steinbichler TB, Kral F, Reinold S, Riechelmann H. Chondrosarcoma of the nasal cavity in a patient with Maffucci syndrome: case report and review of the literature. World Journal of Surgical Oncology 2014;12(1):1-4. 
9. Lenny Dang, David W White, Stefan Gross, Bryson D Bennett, Mark A Bittinger, Edward M Driggers, et al. Cancer-associated IDH1 mutations produce 2-hydroxyglutarate. Nature 2010;462(7274):739-44.

10. Yang Z, Jiang B, Wang Y, Ni H, Zhang J, Xia J, et al. 2-HG Inhibits Necroptosis by Stimulating DNMT1-Dependent Hypermethylation of the RIP3 Promoter. Cell Reports 2017;19(9):1846-57.

11. Pope WB, Prins RM, Thomas MA, Nagarajan R, Yen KE, Bittinger MA, et al. Non-invasive detection of 2-hydroxyglutarate and other metabolites in IDH1 mutant glioma patients using magnetic resonance spectroscopy. Journal of Neuro-Oncology 2012;107(1):197-205.

12. Twinkal C Pansuriya, Ronald van Eijk, Pio d'Adamo, Maayke A J H van Ruler, Marieke L Kuijjer, Jan Oosting, et al. Somatic mosaic IDH1 and IDH2 mutations are associated with enchondroma and spindle cell hemangioma in Ollier disease and Maffucci syndrome. Nature Genetics 2011;43(12):1256-61.

13. BratDJ, PachterL. Comprehensive, Integrative Genomic Analysis of Diffuse Lower-Grade Gliomas. 2015

14. Elaine R Mardis, Li Ding, David J Dooling, David E Larson, Michael D McLellan, Ken Chen, et al. Recurring mutations found by sequencing an acute myeloid leukemia genome. N Engl J Med 2009;361(11):1058-66.

15. Papaemmanuil E, Gerstung M, Bullinger L, Gaidzik VI, Paschka P, Roberts ND, et al. Genomic Classification and Prognosis in Acute Myeloid Leukemia. N Engl J Med 2016;374(23):2209-21.

16. Hao S, Hong CS, Jie F, Yang C, Zhuang Z. Somatic IDH1 mutation in a pituitary adenoma of a patient with Maffucci syndrome. Journal of Neurosurgery 2015;124(6):1.

17. Takahide Nejo, Shota Tanaka, Masako Ikemura, et al. Maffucci syndrome complicated by three different central nervous system tumors sharing an IDH1 R132C mutation: case report. J Neurosurg $2019 ; 131(6): 1829-34$.

18. Schwartz HS, Zimmerman NB, Simon MA, Wroble RR, Millar EA, Bonfiglio M. The malignant potential of enchondromatosis. Jbone Joint Surga 1987;69(2):269. I

19. Dan R, Popovici-Muller J, Palaskas N, Turcan S, Grommes C, Campos C, et al. An Inhibitor of Mutant IDH1 Delays Growth and Promotes Differentiation of Glioma Cells. Science,340.

20. Wang F, Travins J, arre BDa, Penard-Lacronique V, Schalm S, Hansen E, et al. Targeted Inhibition of Mutant IDH2 in Leukemia Cells Induces Cellular Differentiation. Science 2013;340.

\section{Figures}




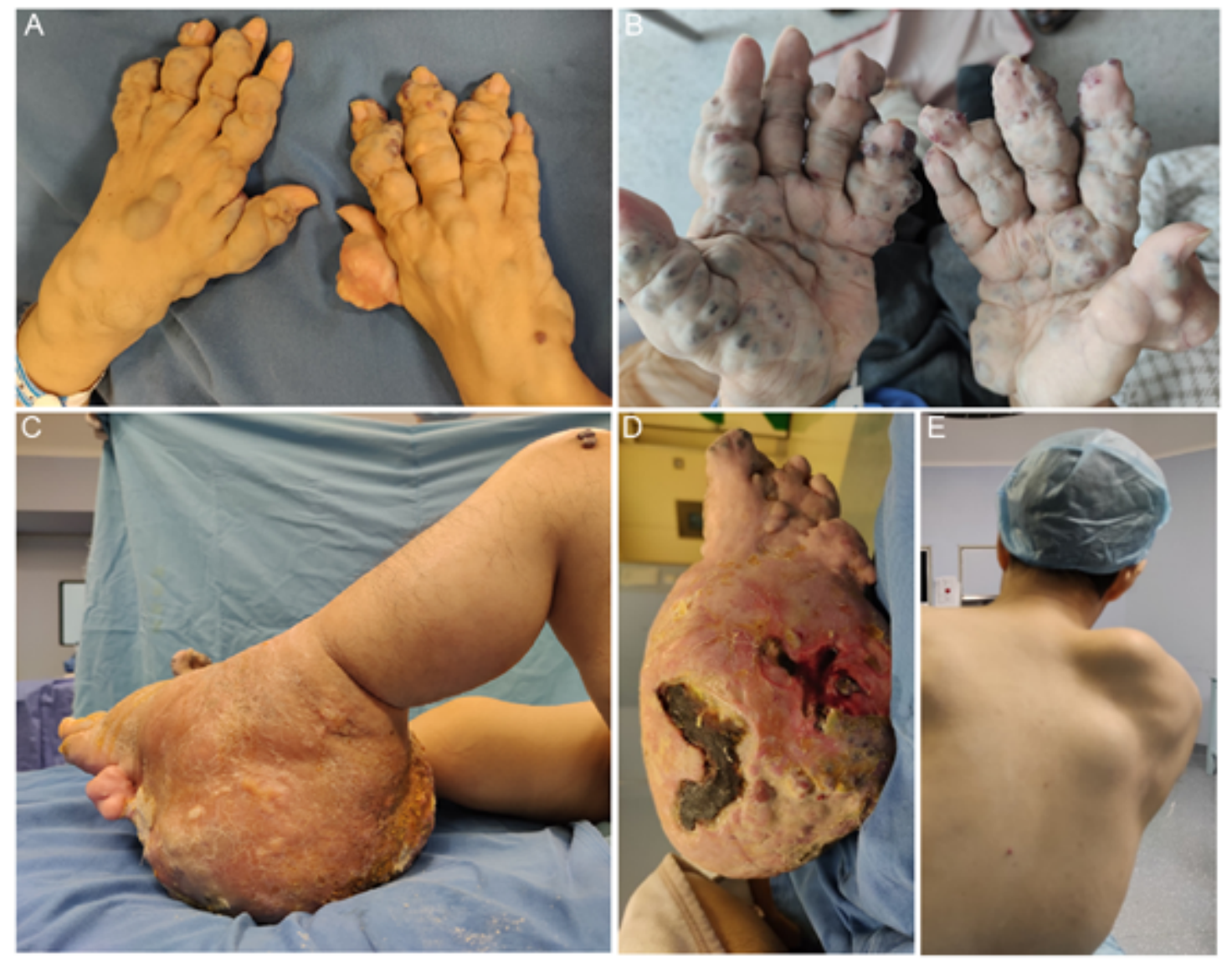

\section{Figure 1}

Physical examination. (A-B) Multiple angiomatoses and enchondromas in the extremities, (C-D) A giant lump in the left ankle, (E) A giant lump on the right scapula. 


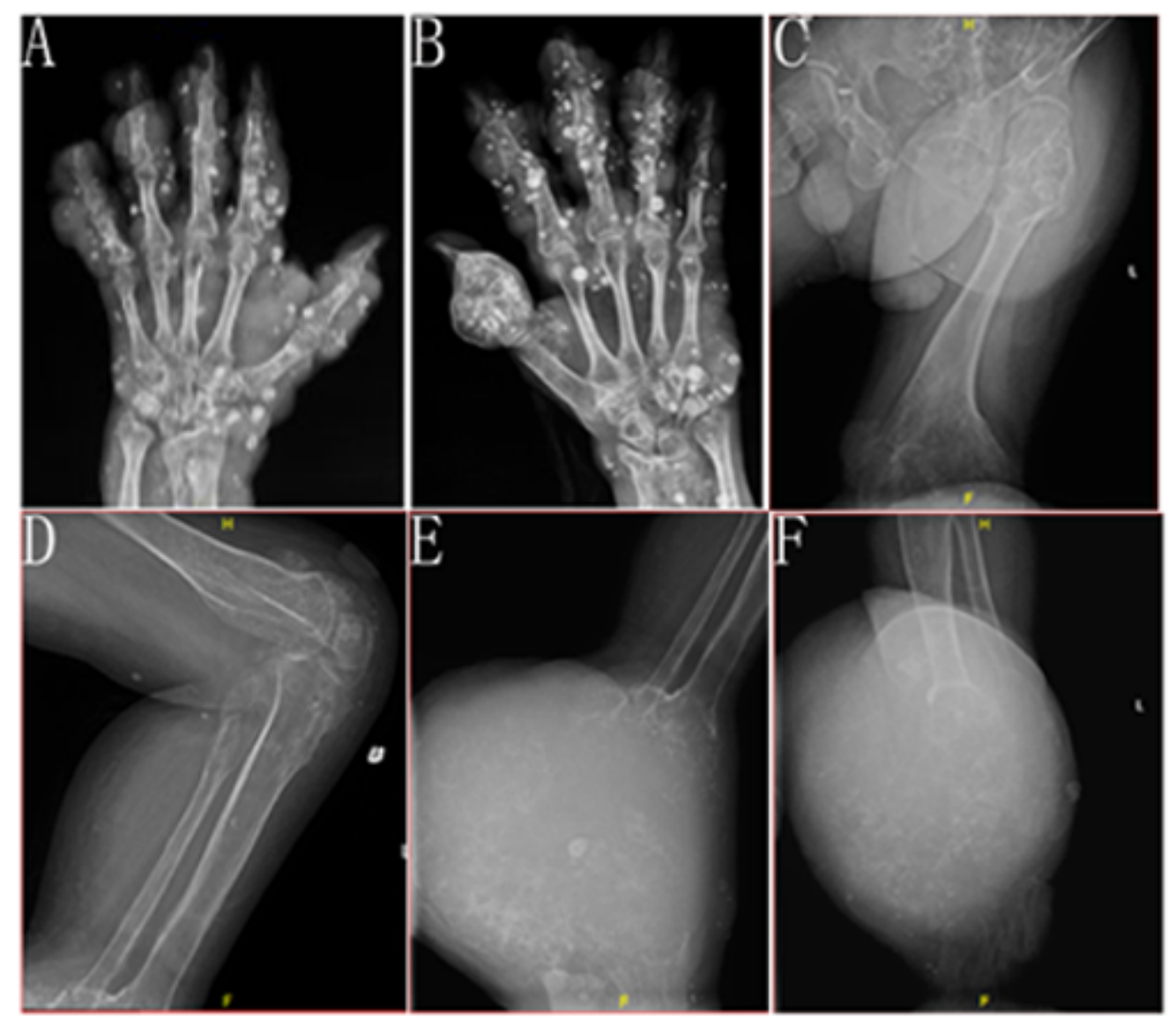

Figure 2

CT examination. (A-B) Spherical expansive bone destruction in the right thumb and multiple phleboliths in the hands and wrists, (C) Bone density and morphological changes of left ilium and left femur, (D) Multiple venous stones around the left knee joint, (E-F) Spherical expansive bone destruction with multiple calcifications at the left tarsal. 


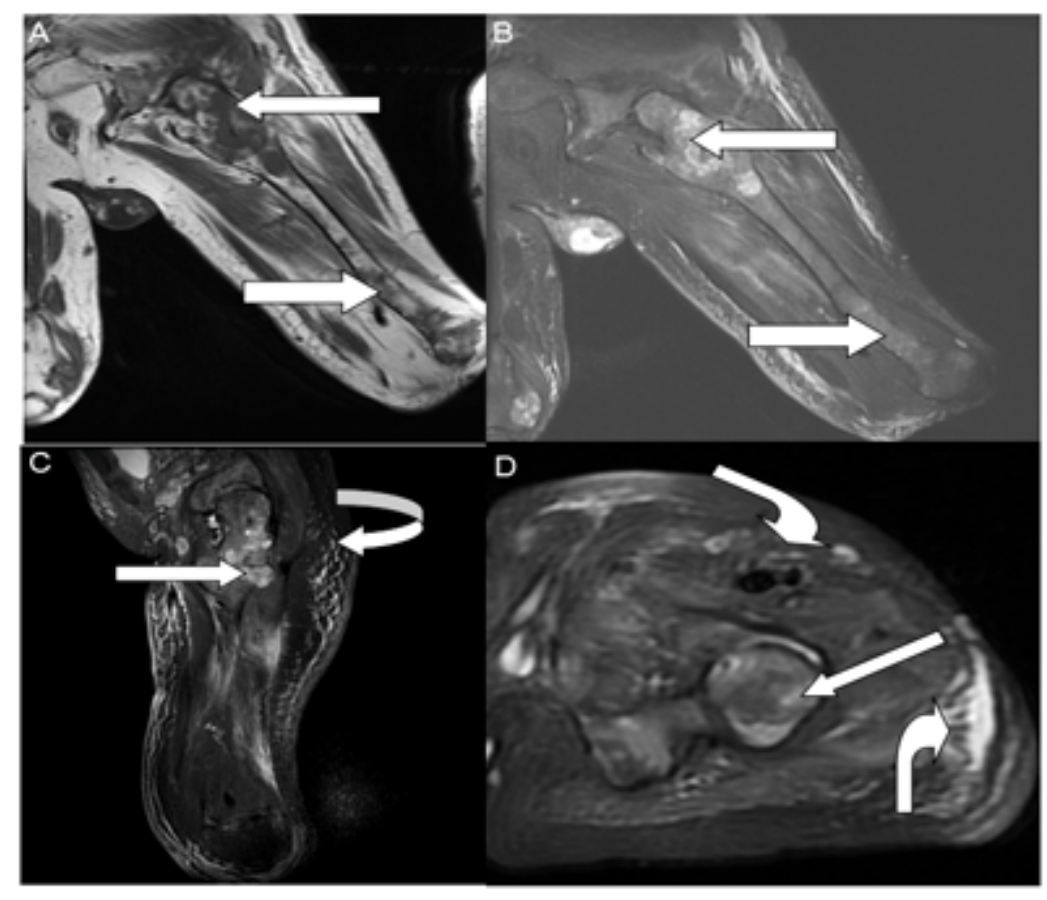

Figure 3

Preoperative imaging studies. (A) low signal on T1WI, (B-D) STIR showed inhomogeneous high signal with unclear boundary and adjacent cortical destruction.
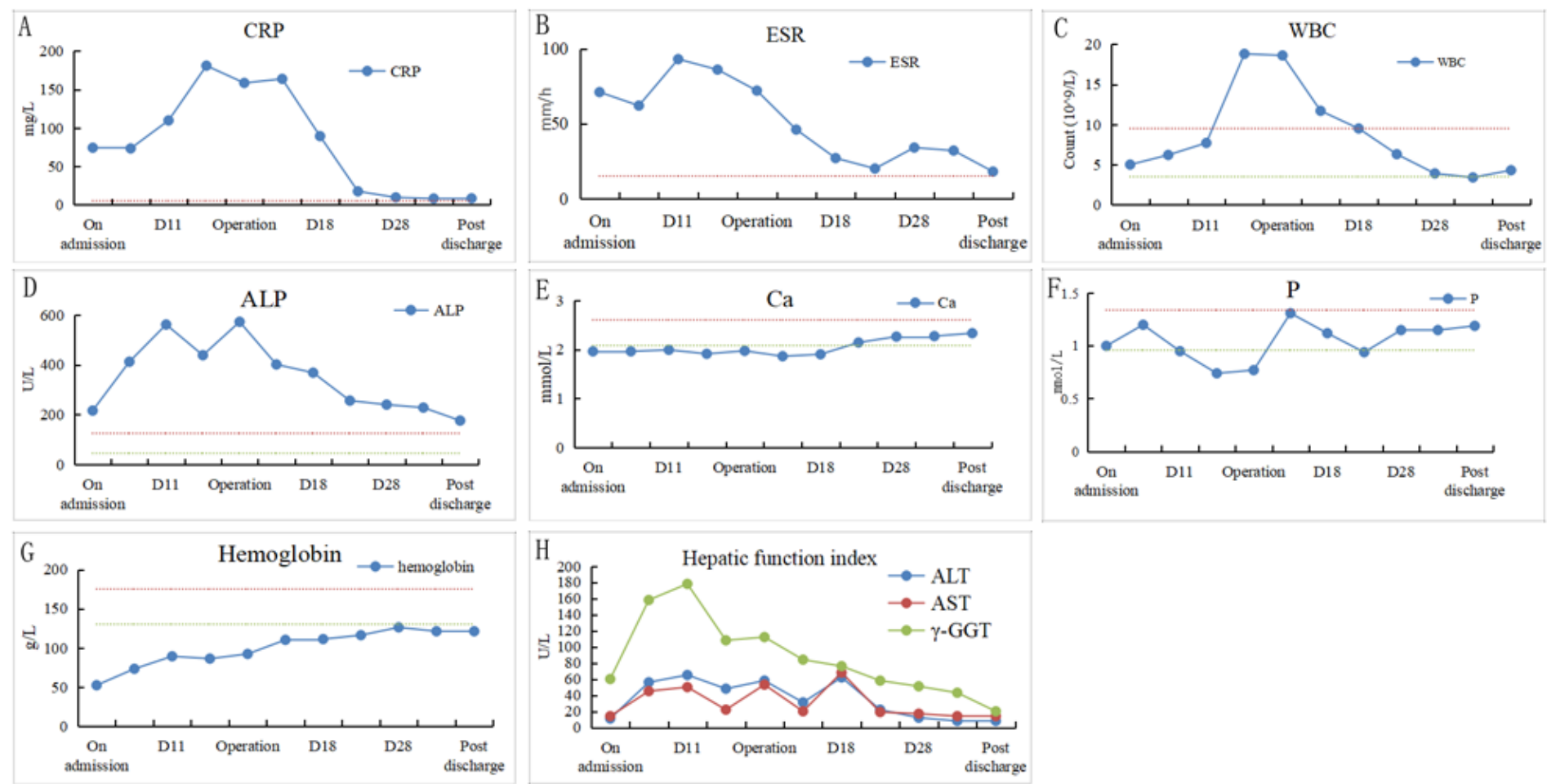

\section{Figure 4}

The dynamics of laboratory variables at admission, discharge, 2nd follow-up. Blue and red dashes indicate the lower and upper values of the normal reference interval. 

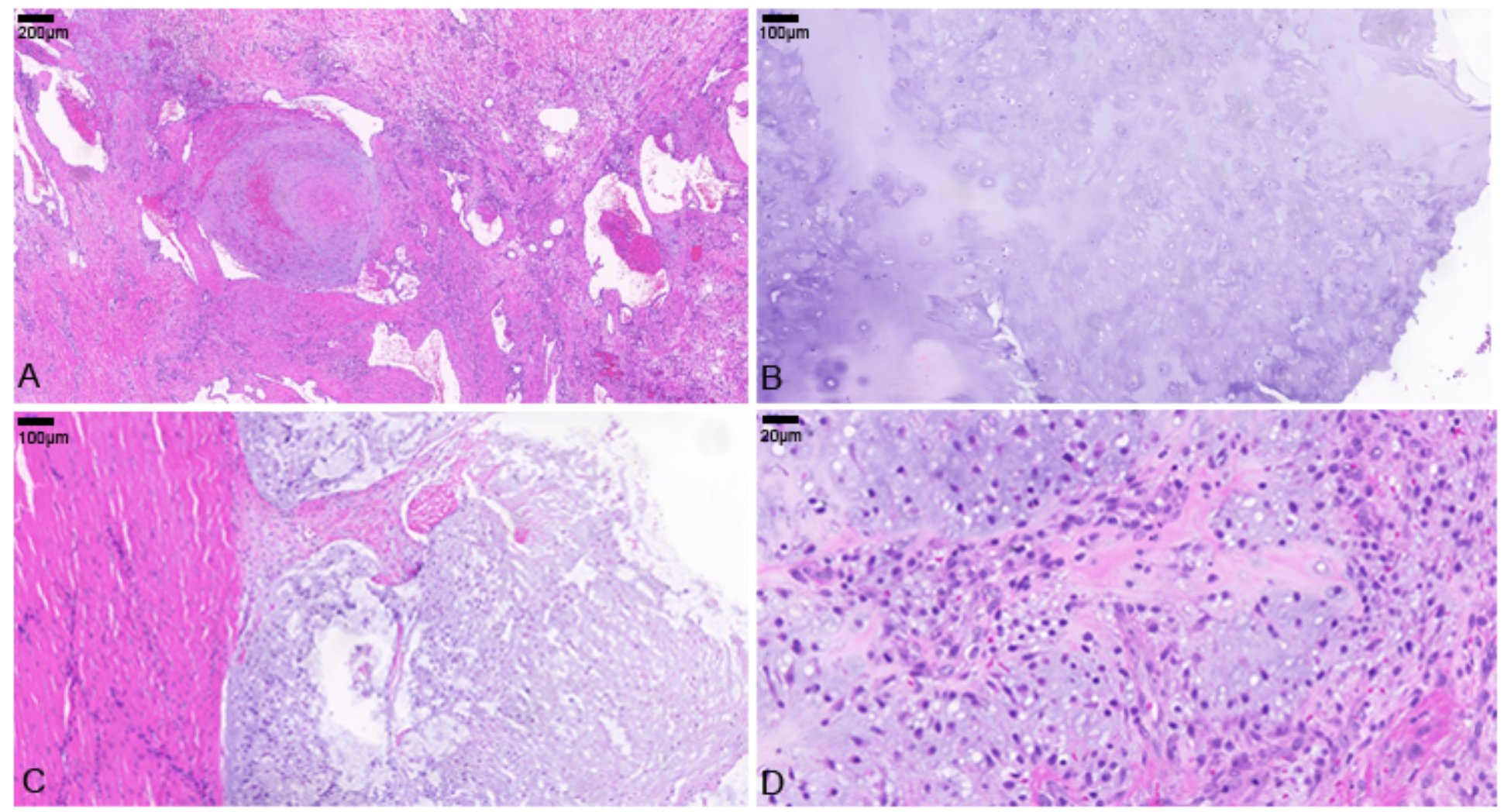

Figure 5

Microscopical features of the pathological sections with hematoxylin-eosin staining. (A) Hemangioma, $\times 5$, (B) Endochondroma, $\times 10,(C-D)$ Chondrosarcoma, $\times 10, \times 40$, respectively. 